\title{
The accurate assessment and physiotherapeutic treatment of Rotator Cuff Myofascial Pain Syndrome: A case report
}

\begin{abstract}
Management of patients with rotator cuff myofascial pain syndrome varies and successful intervention is dependent on accurate assessment. The aim of this case report is to show the importance of accurate assessment and clinical reasoning in the physiotherapeutic management of a patient suffering from ante-cubital and anterior shoulder pain. The patient was referred for physiotherapy after proving refractory to treatment with non-steroidal anti-inflammatory medication. The physiotherapist diagnosed a

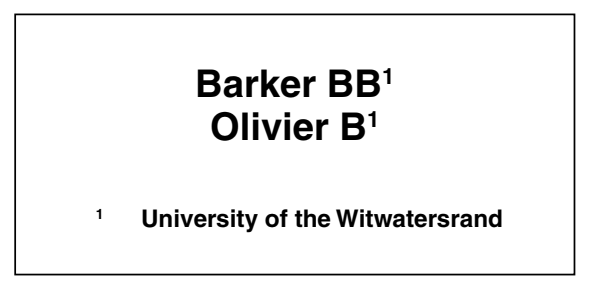
rotator cuff myofascial pain syndrome and treatment proceeded on that basis. Treatment consisted of twitch-obtaining dry needling, myofascial release and exercise therapy. The result was a change in the Harryman Rotator Cuff Functional Assessment Scale score from 22/52 to 43/52 over eight treatments. Strength was regained and subjective pain report on the visual rating scale was improved to 1/10. The case study highlights the importance of accurate assessment and consideration of alternative myofascial sources for pain even in circumstances which initially seem trauma related. Precise diagnosis of the cause - in this case rotator cuff myofascial pain syndrome - will result in effective treatment being administered.
\end{abstract}

KEY WORDS: MYOFASCIAL PAIN SYNDROME, TRIGGER POINTS, INFRASPINATUS, BICEPS BRACHII.

\section{INTRODUCTION}

Physiotherapeutic assessment of musculoskeletal pain includes an in-depth interview, accurate assessment of strength and movement, the performance of special tests and palpation. Higgs et al. (2008:236) defined clinical reasoning as "the cognitive processes and strategies used to understand the significance of patient data, to identify and diagnose actual or potential patient problems, to make clinical decisions to assist in problem resolution, and to achieve positive patient outcomes." Based on an accurate assessment and sound clinical reasoning, the physiotherapeutic management plan is developed. The importance of the assessment and clinical reasoning process is thus emphasised, as this will influence the nature of the physiotherapeutic management plan developed.

The reflective analysis of the signs and symptoms identified during the assessment will lead to the generation of a variety of possible diagnoses. Pain in the ante-cubital fossa is often diagnosed at face value as bicipital tendon injury
(Brukner and Khan 2007), but there may also be alternative diagnoses. In this case it is important to consider other causes of ante-cubital pain as well. Ante-cubital pain may be equally derived from the referral of myofascial trigger points in the biceps brachii and infraspinatus muscles (Travell and Simons 1999). Careful and accurate assessment of these muscles may elicit the comparable pain. Muscle length and strength testing, as well as palpation may indicate the presence of trigger points (Travell and Simons 1999).

Dry needling has proposed to be an effective modality in the treatment of myofascial trigger points (Hong and Chen 1997; Cummings and White 2001). The effectiveness of treatment increased when dry needling is used in combination with other modalities such as stretching (Edwards and Knowles 2003). Included in the treatment of myofascial pain, the use of positional release (Chaitow 2001), shoulder proprioception and stabilising exercises (Lephart et al. 1997) have proposed to be effective.
The aim of this case report is to show the importance of accurate assessment and clinical reasoning in the physiotherapeutic management of a patient suffering from ante-cubital and anterior shoulder pain. Accurate diagnosis of the cause - in this case rotator cuff myofascial pain syndrome - resulted in effective treatment being administered.

\section{CASE DESCRIPTION}

\section{Assessment}

$\mathrm{Mr} \mathrm{X}$ is a $1.76 \mathrm{~m}$ tall, 34 year-old drillingrig operator in an underground trackless mine. He reported a 10 week history of pain in the right ante-cubital fossa (A1)

Corresponding author:

Bruce Barker

Physiotherapy Department

University of the Witwatersrand

Johannesburg

Gauteng

Email: barkerbb@gmail.com 
and anterior aspect of the right shoulder (A2) when training his upper limbs in his chosen sport (bodybuilding), and when performing sudden movements with the right arm ("swatting a fly"). He was particularly distressed by the pain when lifting his two-year old son. Despite the physical nature of this job, he did not suffer pain at work. Initial use of non-steroidal anti-inflammatory medication had little effect on the pain. Sonographic examinations of his right shoulder and right elbow revealed no pathology. He could identify no definite single episode of direct trauma to the arm, but thought the onset of the problem related to a particularly intense training session using the "Pec Deck" resistance training machine immediately followed by an intense session using $30 \mathrm{~kg}$ dumbbells ("inside curls").

The patient's presenting ante-cubital pain $(\mathrm{A} 1=10 / 10)$ was his main concern, and the anterior shoulder pain (A2 = $3 / 10$ ) was described as arising from $A 1$ (refer to figure 1). A1 was eased to 2/10 within seconds by the patient actively shaking the arm by his own volition (ie not as part of therapy). The patient had only been training for six months off a very low fitness base before onset of pain. He had been training with a more experienced partner and was by his own admission training beyond his abilities. Both upper limbs were significantly hypertrophied and appeared to be symmetrical on observation. The scapulae were held in an anterior-inferior glenoid presentation bilaterally.

Clearing tests of the neck and wrist areas were both negative. The right shoulder had full range of active movement but demonstrated a painful arc (A2) during a scaption movement (elevation in a plane $30^{\circ}$ anterior from abduction). The "empty can" test was positive (A1 and A2) (Brukner and Khan 2007). Speed's test for bicipital tendonitis was negative (Brukner and Khan 2007). Palpation of the Infraspinatus muscle reproduced his pain (A1 and A2 simultaneously) exactly, but the length test for Infraspinatus (Hand-behindback-to-T12 test) was not positive, and neither did the patient have pain on recovering from the Hand-behind-back position to anatomical neutral (Brukner

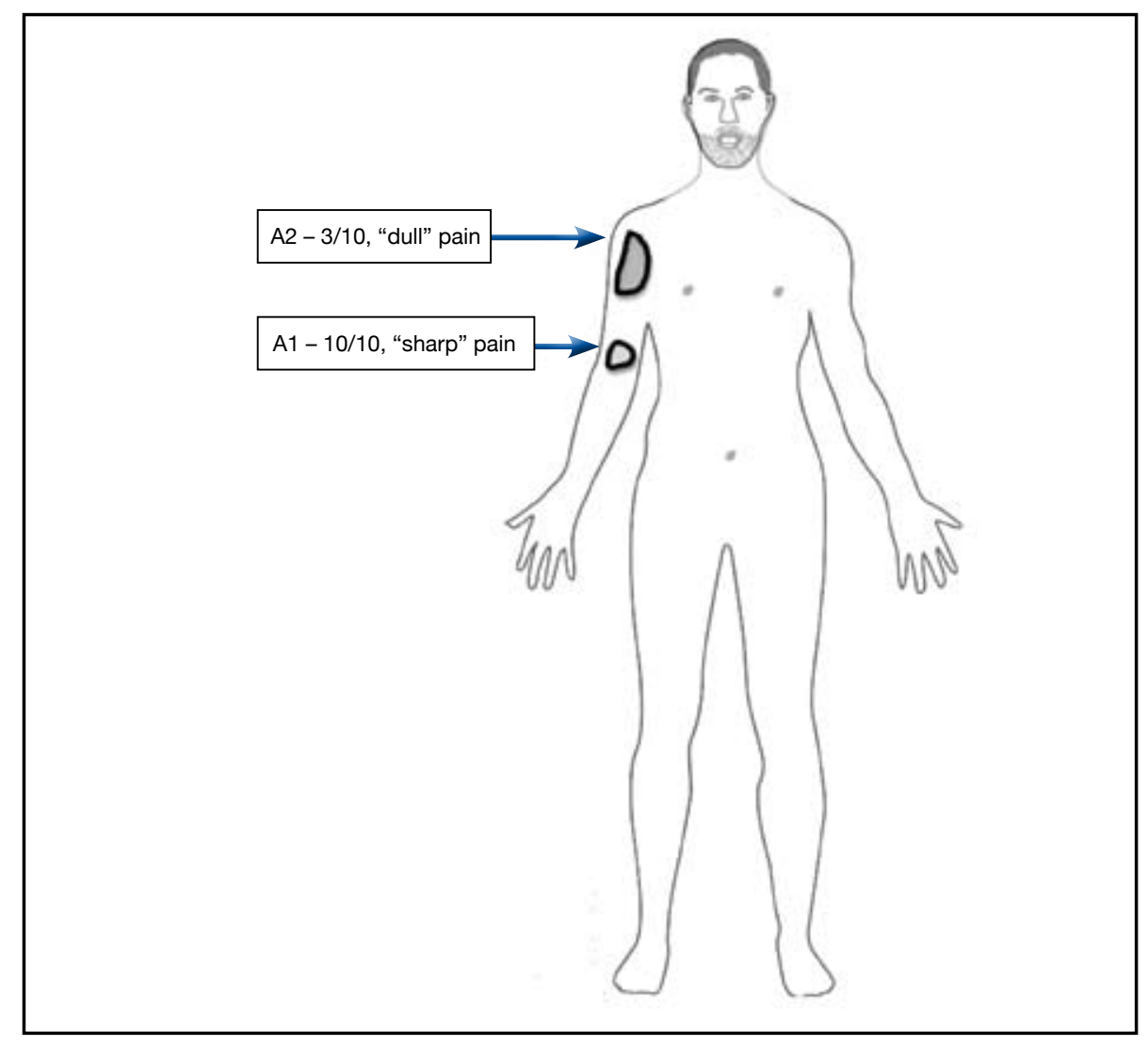

Figure 1: Body chart of Mr X

and Khan 2007). Palpation of the right Supraspinatus muscle revealed local tenderness with moderate familiar pain (A2). Palpation of the Biceps Brachii muscle proved to be tender but did not elicit the familiar pain. There was no instability in either the glenohumeral or humero-ulnar joints when tested with the load and shift and the posterolateral rotatory-instability tests, respectively. Upper limb neural provocation test 1 (median nerve bias) was bilaterally symmetrical and negative. Lateral rotation and elevation shoulder strength was measured at $30 \%$ of the patient's maximal strength with pain being the limiting factor rather than inability to recruit the muscle. The patient was evaluated on a Harryman Rotator Cuff Functional Assessment Scale where he initially scored 22/52 (Harryman et al 1991). Refer to figure 2 for more information on this patient self report assessment tool.

\section{Clinical reasoning}

The patient was diagnosed with right sided rotator cuff myofascial pain syndrome based on four elements:
1. A sub-acute history of overuse, overstrain and overload of the upper limb in toto but with an acute emphasis on outer range eccentric loading of the posterior cuff.

2. The patient's pain recognition (A1 and A2) when palpating the Infraspinatus muscle on the right with confirmatory familiar referral of pain to the ante-cubital fossa (A1). Referral of pain to the ante-cubital fossa is a typical area of referral for trigger points in the Infraspinatus muscle (Travell and Simons 1999). This picture was clouded by the fact that Infraspinatus length testing did not elicit the comparable pain. There was also tenderness on palpation of the Biceps Brachii muscle on the right, but not as severe as palpation of the Infraspinatus muscle.

3. Negative sonographic evidence of ruptures which accord with the lack of a history of bruising in the involved areas, and the grossly symmetrical anatomy.

4. No evidence neurodynamic or neurological limitation was found in the assessment. 
Type: Patient reported outcome

\section{3 items related to the following actions (each item scored on a four point scale):}
1. Reach into back pocket
2. Wash opposite axilla
3. Comb hair
4. Use hand overhead
5. Use extremity to pull something
6. Use arm to dress
7. Sleep on affected side
8. Wash perineum
9. Eat with a utensil
10. Reach out elbow at shoulder level
11. Carry weight at side
12. Lift a weight
13. Throw a ball

Score: Maximum $=52$, Minimum $=0$

\section{Management}

Therapy was aimed at reducing pain at A1 and A2 during sudden movements and when lifting heavy loads. Particular emphasis was laid at the facilitation of normal scapular biomechanics. A combination of myofascial therapies including twitch-obtaining dry needling and positional release, stretching and stabilising exercises was employed on a total of eight occasions each separated by a gap of between two and four days according to the patient's work schedule. Proprioceptive taping using Kinesiotape ${ }^{\circledR}$ from the Infraspinatus area to the anterior shouder was applied at the end of the treatment session. Only once proximal control of the scapulothoracic and glenohumeral joints was achieved were resisted closed chain exercises added, and then progressed to his normal weight lifting activities.

Dry needling and myofascial release were performed on the right Infraspinatus muscle trigger points (Travell and Simons 1999). The patient had an immediate improvement of $50 \%$ on lateral rotation and elevation strength following the initial needling session during which strong myofascial twitches were noted. There was up to $80 \%$ improvement of strength by the third treatment when the use of needling was discontinued. The patient felt good and subsequently decided to participate in strenuous work related special training drills ("Proto team") after the third treatment. By treatment five (day 14) the patient reported a $90 \%$ improvement in the initial pain on the visual rating scale. Treatment was discontinued after treatment eight (day 23) with a Harryman Rotator Cuff Functional Assessment Scale score of $43 / 52$ and a $100 \%$ return of lateral rotation and elevation strength.

\section{OUTCOME}

The patient achieved a good outcome. The Harryman Rotator Cuff Functional Assessment Scale scores improved from $22 / 52$, to $43 / 52$ on discharge. There was almost complete resolution of the subjective arc of pain A2 (1/10) and ability to sleep on the right side. Shoulder lateral rotation and elevation strength was returned. A1 was abolished. The "empty can" test was negative (A1 and A2) and palpation of Infraspinatus did not reproduce his pain any longer. The patient was able to play with his child again without pain.

\section{DISCUSSION}

The accurate assessment of a patient as described in this case report proved to be of utmost importance. Pain in the ante-cubital fossa is commonly associated with bicipital tendon injury or elbow overuse injury. Trigger points in the biceps brachii muscle however also refers to the anterior shoulder as well as to the ante-cubital fossa. If premature closure in the clinical reasoning process took place at this stage it could have led to misdiagnosing this patient. Trigger points in the Infraspinatus muscle may refer pain to the anterior shoulder, over the biceps brachii area to the antecubital fossa. In this case, palpation of the biceps brachii muscle, although tender, was not as tender as palpation of the Infraspinatus muscle proved to be. The palpation of the Infraspinatus muscle trigger points also elicited the comparable pain (A1 and A2), while palpation of the biceps brachii muscle trigger points did not. The pain referral pattern of the Infraspinatus muscle trigger points may have been responsible for secondary trigger points in the biceps brachii muscle, causing the resultant tenderness. The empty can test may have been positive initially as $\mathrm{Mr} \mathrm{X}$ may have recruited his Infraspinatus muscle to act as a stabiliser during this test. The link between myofascial trigger points in the Infraspinatus muscle and ante-cubital fossa pain is not always made during the assessment.

As a result of an accurate assessment done - a combination of structural integrity testing, palpation and clinical reasoning - an effective treatment programme could be structured. In a systematic review of needling therapies in the treatment of myofascial trigger points, direct dry needling appears to be an effective treatment of myofascial pain and dysfunction (Cummings and White 2001). Dry needling appears to be more effective than digital palpation of myofascial trigger points in eliciting the characteristic referred pain (Hong and Chen 1997). In a single-blind, randomised control trial, UK physiotherapists Edwards and Knowles (2003) 
have conclusively shown that dry needling combined with therapeutic stretching is more effective than stretching alone in reducing pain due to myofascial trigger points. The use of dry needling within a focussed, rehabilitative exercise framework is thus recommended.

\section{SIGNIFICANCE AND CONCLUSION}

This case study shows the importance of accurate initial assessment as well as the ensuing physiotherapeutic management. The patient complained of a debilitating A1 (10/10) and its associated A2 (3/10) pain for 10 weeks before physiotherapeutic treatment commenced. After just three sessions over a 10 day period, he performed functional activities with minimal pain. This case study adds to the current understanding of upper limb pain by underscoring the importance of accurate assessment, concise clinical reasoning, and effective treatment of the myofascial elements of pain and dysfunction.

\section{ETHICAL CONSIDERATIONS}

Signed informed consent was obtained from the patient for all aspects of therapy and the use of information contained in this report.

\section{REFERENCES}

Brukner P, and Khan K, 2007 Clinical Sports Medicine Third Edition, McGraw-Hill: Australia

Chaitow L, 2001 Positional release techniques Second edition, Churchill Livingstone: Oxford

Cummings T, and White AR, 2001 Needling Therapies in the Management of Myofascial Trigger Point Pain. Archives of Physical Medicine and Rehabilitation 82:986-992

Edwards J, Knowles N, 2003 Superficial Dry Needling and Active Stretching in the Treatment of Myofascial Pain: A randomised control study. Acupuncture in Medicine 21: 80-86

Harryman DT 2 $2^{\text {nd }}$, Mack LA, Wang KY, Jackins SE, Richardson ML, Matsen FA 3rd 1991 Repairs of the rotator cuff. Correlation of functional results with integrity of the cuff. Journal of Bone and Joint Surgery of America 73:982-989

Higgs J, Jones MA, Loftus S, Christensen N, 2008 Clinical reasoning in the health professions Third edition, Elsevier, Amsterdam

Hong C-Z, Kuan T-S, Chen J-T, Chen S-M, 1997 Referred Pain elicited by Palpation and by Needling of Myofascial Trigger Points: A Comparison. Archives of Physical Medicine and Rehabilitation 78:957-960
Kolber MJ, and Cleland JA, 2005 Strength Testing Using Hand Held Dynamometry. Physical Therapy Reviews 10:99-112

Lephart SM, Pincivero DM, Giraido JL, Fu FH, 1997 The Role of Proprioception in the Management and Rehabilitation of Athletic Injuries, American Journal of Sports Medicine 25:130-137

Travell JG, Simons DG, Simons LS, 1999 Myofascial Pain and Dysfunction: The Trigger Point Manual. Volume 1. 2ed. Williams \& Wilkins, Baltimore 